\title{
Periodontal Bacteria and Epithelial Cell Interactions: Role of Bacterial Proteins
}

Periodontal diseases are a group of chronic multifactorial infections that initiate destruction of periodontium, the tissues that surround and support the teeth. The main etiological agent of periodontitis is the microbial dental plaque. Etiologically most important periodontal microorganisms are gram-negative, facultative or obligatory anaerobic rods; the strongest association between periodontitis and a pathogen is provided by Aggregatibacter actinomycetemcomitans, Porphyromonas gingivalis, Tannerella forsythia, Prevotalla intermedia, and Fusobacterium nucleatum, but several other species also have potential pathogenicity. Potential virulence factors of periodontal pathogens include proteolytic enzymes, leukotoxin, endotoxin (lipopolysaccharide (LPS) of gram-negative bacteria), evasion of host responses, invasion to host tissues, and induction of inflammatory mediators, such as interleukin (IL)-1, IL-6, IL-8, and tumor necrosis factor- alpha (TNF- $\alpha$ ). ${ }^{1-3}$ However, it is still unclear what role the different virulence factors play in the different phases of infection.

Specific periodontopathogens are capable of attaching to and invading periodontal cells, notably gingival epithelial cells. $A$. actinomycetemcomitans, $P$. gingivalis, $P$. intermedia, and $F$. nucleatum have been observed to attach and invade host epithelial cells in vitro. Electron microscopy and culture studies demonstrated that $P$. gingivalis adheres to and internalizes within primary and multilayer cultures of gingival epithelial cells. Protein synthesis was found to be necessary for its invasion, and about $90 \mathrm{~min}$ is required to complete the invasion process for most of the bacterial cells. P. gingivalis can also spread directly from one epithelial cell to the other, by taking the advantage of host cell actin cytoskeleton. Invasion of $A$. actinomycetemcomitans to epithelial cells demands activity of both keratinocyte and the bacteria and the attachment of $A$. actinomycetemcomitans to epithelial cells requires fimbriae and/or extracellular vesicles. Immediately after its entry, A. actinomycetemcomitans undergoes rapid multiplication and direct itself to host cell protrusions, which may mediate cell-to-cell spread of the bacteria. F. nucleatum is among the most numerous bacterial species in dental plaque and it is also believed to play an important role in the pathogenesis of periodontitis. We have recently demonstrated that it can also invade epithelial cells and multiply intracellularly. Prevotella species are common oral anaerobes that can be isolated even from early childhood. In our latest research, we showed that the $P$. intermedia and $P$. nigrescens seem to adhere and invade epithelial cells in a strain-dependent manner and the adhesion of $P$. intermedia to epithelial cells occur via site-specific binding ability of bacteria to host cell lamellipodia. ${ }^{3-6}$

Proteomics allows determination of the level of protein expression, both in eukaryotic and prokaryotic cells. The method can be used to examine protein-protein interactions, to determine the presence of protein isoforms and post-translational modifications. There are several different methods available to evaluate proteomes of bacterial cells; however Two-dimensional gel electrophoresis (2-DE) has been one common method to study proteomics of several oral bacteria. 2-DE allows visualization of hundreds of proteins at a time. Since surface proteins are closely related to adhesion capability of bacteria, initial proteomic studies have been focused on outer membranes of periodontal pathogens. Thirty-nine outer membrane proteins have been identified from $P$. gingivalis with 2-DE, and several of those proteins showed $\mathrm{pl}$ heterogeneity that was observed as a train of horizontal spots. When $P$. gingivalis was exposed to epithelial cell extracts, 479 proteins (total 1014) showed evidence of 
differential expression. This result suggests that the adaptation of bacteria to an epithelial cell environment induces a major shift in the expressed bacterial proteome. Proteomic studies also identified a $17 \mathrm{kDa}$ A. actinomycetemcomitans protein as a peptidoglycan-associated protein with subsequent sequence analysis with mass spectrophotometry. This protein was found to be a strongly immunoreactive antigen in patients with periodontitis. ${ }^{7,8}$

In understanding the bacterial virulence mechanisms, clarification of the behavior of bacteria in various phases of their relationship with host cells is essential. In this respect, several different conditions mimicking host cell - bacterial cell interactions and proteomic analyses of intracellular and extracellular proteins need to be used to investigate bacterial protein expression related to periodontal infections. In order to understand the bacterial virulence mechanism in whole, it is essential to know about protein expression patterns of both bacteria and host cells in their interactions. Intracellular survival of bacteria gives them an advantage of adapting to several rough conditions, including resistance to host defense and antibiotics. These studies hopefully will be directed to analyze the adaptation capabilities of clinical bacteria and overcome their resistance to medications, especially antibiotics.

\section{REFERENCES}

1. Socransky SS, Haffajee AD. Periodontal microbial ecology. Periodontol 2000 2005;38:135-187.

2. Gursoy UK, Könönen E, Uitto VJ. Stimulation of epithelial cell matrix metalloproteinase (MMP-2, -9, -13) and interleukin-8 secretion by fusobacteria. Oral Microbiol Immunol 2008;23:432-434.

3. Uitto VJ, Baillie D, Wu Q, Gendron R, Grenier D, Putnins EE, Kanervo A, Firth JD. Fusobacterium nucleatum increases collagenase 3 production and migration of epithelial cells. Infect Immun 2005;73:1171-1179.

4. Könönen E, Wolf J, Mättö J, Frandsen EVG, Poulsen K, Jousimies-Somer H, Asikainen S. The Prevotella intermedia group organisms in young children and their mothers as related to maternal periodontal status. $J$ Periodontal Res 2000;35:329-334.

5. Yilmaz O, Verbeke P, Lamont RJ, Qjcius DM. Intercellular spreading of Porphyromonas gingivalis infection in primary gingival epithelial cells. Infect Immun 2006;74:730-710.
6. Meyer DH, Lippmann JE, Fives-Taylor PM. Invasion of epithelial cells by Actinobacillus actinomycetemcomitans: a dynamic, multistep process. Infect Immunity 1996;64:29882997.

7. Macarthur DJ, Jacques NA. Proteome analysis of oral pathogens. J Dent Res 2003;82:870-876.

8. Paul-Satyaseela M, Karched M, Bian Z, Ihalin R, Boren T, Arnqvist A, Chen C, Asikainen S. Immunoproteomics of Actinobacillus actinomycetemcomitans outer-membrane proteins reveal a highly immunoreactive peptidoglycanassociated lipoprotein. J Med Microbiol 2006;55:931-942. 